\title{
Misunderstanding Nonlinear Prices: Evidence from a Natural Experiment on \\ Residential Electricity Demand
}

\section{APPENDIX}

\section{A1. Balanced dataset preparation}

From the raw data, I perform the following operations to prepare a suitable dataset for analysis. First, I consider only households with continuous bill data for the entire duration of the 2005-2013 period. This cuts the number of valid observations significantly, as many premise IDs include only sporadic readings. While this cuts the power of the empirical analysis, I have little reason to believe the remaining premises are systematically different than omitted accounts. Second, I exclude any households with negative consumption values or values that exceed ten times the median bill. Extreme values such as these occur on occasion due to meter misreads or corrections. Excluding households containing these extreme values eliminates less than 300 households. Finally, the individual household bills are not of uniform length, nor do they all follow the same cycle. To deal with the non-uniform length, I calculate a dailykwh variable as the total amount consumed divided by the (different) days in each billing cycle. To deal with different billing cycle start/end dates, I use the dailykwh variable to create a daily value for each day in the 2005-2013 period and subsequently collapse the daily data back to calendar months. This creates a balanced dataset with standard periods of length and aligned cycles. The number of households and total observations in the balanced dataset are reduced to 34,592 and 3.7 million, respectively. 


\section{A2. Derivation of consumer surplus}

We start with the assumption of consumers optimizing based on a quasilinear utility function of the following form:

$$
\max _{X, Q} U(X, Q)=X+\alpha Q^{\beta} \text { subject to } I=X+\tilde{P} Q
$$

where $X$ is the composite good, $Q$ is electricity, and $\tilde{P}$ is the perceived price of electricity. The first order conditions deliver the following demand function for electricity:

$$
Q=\left(\frac{\tilde{P}}{\alpha \beta}\right)^{\frac{1}{\beta-1}}
$$

Taking $\operatorname{logs}$ and letting $\epsilon=\frac{1}{\beta-1}$ gives:

$$
\ln Q=-\alpha \ln (\alpha \beta)+\epsilon \ln \tilde{P} .
$$

Differentiating with respect to $\ln \tilde{P}$ gives the price elasticity of demand, $\epsilon$ :

$$
\epsilon=\frac{d \ln Q}{d \ln \tilde{P}}=\frac{\% \Delta Q}{\% \Delta \tilde{P}}
$$

We can now use this constant log elasticity in our estimate of the deadweight loss, for which we use the Harberger triangle approximation:

$$
D W L=\frac{1}{2} \Delta Q \Delta \tilde{P}
$$

Noting that for small changes, $\Delta Q$ and $\Delta \tilde{P}$ can be approximated by:

$$
\begin{aligned}
\Delta Q & =\% \Delta Z \cdot Z \\
\Delta P & =\% \Delta \tilde{P} \cdot P \\
& =\frac{\% \Delta Q}{\epsilon} P .
\end{aligned}
$$

The deadweight loss is thus:

$$
\begin{aligned}
D W L & =\frac{1}{2}(\% \Delta Q \cdot Q)\left(\% \Delta Q \frac{1}{\epsilon} P\right) \\
& =\frac{1}{2} \frac{1}{\epsilon} P Q(\% \Delta Q)^{2} .
\end{aligned}
$$


A3. Tables and Figures

Table A1-: Descriptive Statistics By Decile

\begin{tabular}{|c|c|c|c|c|c|c|c|c|c|c|c|c|}
\hline \multicolumn{13}{|c|}{ BC Hydro (all 6 NEIGHBouring FSAs) } \\
\hline & \multicolumn{6}{|c|}{ Demand (kWh) } & \multicolumn{6}{|c|}{ Prices (cents/kWh) } \\
\hline & \multicolumn{3}{|c|}{ Mean } & \multicolumn{3}{|c|}{ Median } & \multicolumn{3}{|c|}{$\mathrm{MC}$} & \multicolumn{3}{|c|}{$\mathrm{AC}$} \\
\hline Decile & Pre & Post & $\Delta$ & Pre & Post & $\Delta$ & Pre & Post & $\Delta$ & Pre & Post & $\Delta$ \\
\hline 1 & 5.9 & 6.6 & $11.2 \%$ & 5.9 & 6.1 & $3.1 \%$ & 6.18 & 6.47 & $4.7 \%$ & 6.18 & 6.44 & $4.1 \%$ \\
\hline 2 & 10.5 & 11.1 & $5.5 \%$ & 10.2 & 10.3 & $1.3 \%$ & 6.18 & 6.52 & $5.4 \%$ & 6.18 & 6.45 & $4.3 \%$ \\
\hline 3 & 14.4 & 15.0 & $4.5 \%$ & 14.0 & 14.2 & $1.1 \%$ & 6.18 & 6.67 & $7.9 \%$ & 6.18 & 6.48 & $4.8 \%$ \\
\hline 4 & 17.9 & 18.3 & $2.4 \%$ & 17.4 & 17.4 & $(0.0 \%)$ & 6.18 & 6.95 & $12.4 \%$ & 6.18 & 6.52 & $5.6 \%$ \\
\hline 5 & 21.4 & 21.8 & $2.1 \%$ & 20.9 & 20.7 & $(0.6 \%)$ & 6.18 & 7.49 & $21.1 \%$ & 6.18 & 6.64 & $7.4 \%$ \\
\hline 6 & 25.1 & 25.2 & $0.6 \%$ & 24.5 & 24.0 & $(2.0 \%)$ & 6.18 & 8.05 & $30.3 \%$ & 6.18 & 6.79 & $9.8 \%$ \\
\hline 7 & 29.1 & 29.0 & $(0.4 \%)$ & 28.4 & 27.6 & $(2.7 \%)$ & 6.18 & 8.49 & $37.3 \%$ & 6.18 & 6.98 & $13.0 \%$ \\
\hline 8 & 34.0 & 33.5 & $(1.5 \%)$ & 33.1 & 31.9 & $(3.7 \%)$ & 6.18 & 8.77 & $41.9 \%$ & 6.18 & 7.20 & $16.5 \%$ \\
\hline 9 & 40.9 & 39.7 & $(2.9 \%)$ & 39.8 & 38.0 & $(4.7 \%)$ & 6.18 & 8.95 & $44.8 \%$ & 6.18 & 7.46 & $20.7 \%$ \\
\hline 10 & 59.3 & 55.6 & $(6.2 \%)$ & 54.4 & 51.0 & $(6.3 \%)$ & 6.18 & 9.01 & $46.8 \%$ & 6.18 & 7.85 & $27.0 \%$ \\
\hline \multicolumn{13}{|c|}{ NeW Westminster } \\
\hline & \multicolumn{6}{|c|}{ Demand $(\mathrm{kWh})$} & \multicolumn{6}{|c|}{ Prices (cents/kWh) } \\
\hline & \multicolumn{3}{|c|}{ Mean } & \multicolumn{3}{|c|}{ Median } & \multicolumn{3}{|c|}{$\mathrm{MC}$} & \multicolumn{3}{|c|}{$\mathrm{AC}$} \\
\hline Decile & Pre & Post & $\Delta$ & Pre & Post & $\Delta$ & Pre & Post & $\Delta$ & Pre & Post & $\Delta$ \\
\hline 1 & 5.9 & 6.5 & $8.5 \%$ & 5.9 & 6.0 & $2.7 \%$ & 6.17 & 7.75 & $25.6 \%$ & 6.17 & 7.75 & $25.6 \%$ \\
\hline 2 & 10.4 & 10.7 & $3.1 \%$ & 10.1 & 10.1 & $0.1 \%$ & 6.17 & 7.75 & $25.6 \%$ & 6.17 & 7.75 & $25.6 \%$ \\
\hline 3 & 14.3 & 14.7 & $2.8 \%$ & 13.8 & 13.8 & $(0.6 \%)$ & 6.17 & 7.75 & $25.6 \%$ & 6.17 & 7.75 & $25.6 \%$ \\
\hline 4 & 17.8 & 18.3 & $2.7 \%$ & 17.1 & 17.0 & $(0.8 \%)$ & 6.17 & 7.75 & $25.6 \%$ & 6.17 & 7.75 & $25.6 \%$ \\
\hline 5 & 21.4 & 21.9 & $2.6 \%$ & 20.6 & 20.6 & $(0.1 \%)$ & 6.17 & 7.75 & $25.6 \%$ & 6.17 & 7.75 & $25.6 \%$ \\
\hline 6 & 25.1 & 25.6 & $2.2 \%$ & 24.2 & 24.1 & $(0.3 \%)$ & 6.17 & 7.75 & $25.6 \%$ & 6.17 & 7.75 & $25.6 \%$ \\
\hline 7 & 29.1 & 29.3 & $0.7 \%$ & 28.3 & 27.8 & $(1.7 \%)$ & 6.17 & 7.75 & $25.6 \%$ & 6.17 & 7.75 & $25.6 \%$ \\
\hline 8 & 34.1 & 33.4 & $(2.0 \%)$ & 33.1 & 32.0 & $(3.4 \%)$ & 6.17 & 7.75 & $25.6 \%$ & 6.17 & 7.75 & $25.6 \%$ \\
\hline 9 & 40.7 & 39.8 & $(2.3 \%)$ & 39.6 & 38.2 & $(3.7 \%)$ & 6.17 & 7.75 & $25.6 \%$ & 6.17 & 7.75 & $25.6 \%$ \\
\hline 10 & 60.5 & 57.2 & $(5.5 \%)$ & 54.6 & 51.5 & $(5.6 \%)$ & 6.17 & 7.75 & $25.6 \%$ & 6.17 & 7.75 & $25.6 \%$ \\
\hline
\end{tabular}

Note: Deciles are determined across all households in the dataset, not separately by region. All statistics relate to the balanced panel dataset. 
Table A2-: CONDitional DifFERENCE-IN-DiffEREnCES ESTIMATES

\begin{tabular}{|c|c|c|c|}
\hline & $(1)$ & $(2)$ & $(3)$ \\
\hline Post\#BCH\#1.decile & $\begin{array}{c}0.0107^{* * *} \\
(0.0024)\end{array}$ & $\begin{array}{c}0.0119 * * * \\
(0.0022)\end{array}$ & $\begin{array}{c}0.0126^{* * *} \\
(0.0020)\end{array}$ \\
\hline Post\#BCH\#2.decile & $\begin{array}{c}0.0200^{* * *} \\
(0.0024)\end{array}$ & $\begin{array}{c}0.0195^{* * *} \\
(0.0023)\end{array}$ & $\begin{array}{c}0.0200^{* * *} \\
(0.0020)\end{array}$ \\
\hline Post\#BCH\#3.decile & $\begin{array}{c}0.0175^{* * *} \\
(0.0025)\end{array}$ & $\begin{array}{c}0.0180 * * * \\
(0.0023)\end{array}$ & $\begin{array}{c}0.0185^{* * *} \\
(0.0021)\end{array}$ \\
\hline Post\#BCH\#4.decile & $\begin{array}{c}0.0099 * * * \\
(0.0026)\end{array}$ & $\begin{array}{c}0.0095^{* * *} \\
(0.0024)\end{array}$ & $\begin{array}{c}0.0100^{* * *} \\
(0.0022)\end{array}$ \\
\hline Post\#BCH\#5.decile & $\begin{array}{c}0.0029 \\
(0.0028)\end{array}$ & $\begin{array}{c}0.0022 \\
(0.0026)\end{array}$ & $\begin{array}{c}0.0028 \\
(0.0023)\end{array}$ \\
\hline Post\#BCH\#6.decile & $\begin{array}{c}-0.0142^{* * *} \\
(0.0029)\end{array}$ & $\begin{array}{c}-0.0151^{* * *} \\
(0.0026)\end{array}$ & $\begin{array}{c}-0.0144^{* * *} \\
(0.0024)\end{array}$ \\
\hline Post\#BCH\#7.decile & $\begin{array}{c}0.0006 \\
(0.0029)\end{array}$ & $\begin{array}{c}0.0001 \\
(0.0027)\end{array}$ & $\begin{array}{c}0.0007 \\
(0.0024)\end{array}$ \\
\hline Post\#BCH\#8.decile & $\begin{array}{c}0.0024 \\
(0.0030)\end{array}$ & $\begin{array}{c}0.0017 \\
(0.0027)\end{array}$ & $\begin{array}{c}0.0024 \\
(0.0024)\end{array}$ \\
\hline Post\#BCH\#9.decile & $\begin{array}{l}-0.0016 \\
(0.0030)\end{array}$ & $\begin{array}{l}-0.0024 \\
(0.0028)\end{array}$ & $\begin{array}{l}-0.0016 \\
(0.0025)\end{array}$ \\
\hline Post\#BCH\#10.decile & $\begin{array}{l}-0.0007 \\
(0.0032)\end{array}$ & $\begin{array}{l}-0.0015 \\
(0.0030)\end{array}$ & $\begin{array}{l}-0.0007 \\
(0.0026)\end{array}$ \\
\hline Year-month FE & $x$ & - & $x$ \\
\hline Household FE & - & $\times$ & $\times$ \\
\hline Observations & $3,721,963$ & $3,721,963$ & $3,721,963$ \\
\hline R-squared & 0.7606 & 0.7969 & 0.7780 \\
\hline Number of households & 34,591 & 34,591 & 34,591 \\
\hline
\end{tabular}

Note: Standard errors (clustered at premise level) shown in parentheses. 
Table A3-: Bunching estimates AT Higher order POlynOmials

\begin{tabular}{lcc}
\hline \hline Polynomial order & Estimate & Standard Error \\
7 & -0.048 & $(0.010)$ \\
8 & -0.045 & $(0.011)$ \\
9 & -0.043 & $(0.011)$ \\
10 & -0.040 & $(0.013)$ \\
11 & -0.041 & $(0.012)$ \\
12 & -0.038 & $(0.013)$ \\
\hline
\end{tabular}

Note: Bootstrapped standard errors shown in parentheses.

Table A4-: Bunching Estimates using Simulated Distributions

\begin{tabular}{lccccc}
\hline \hline Counterfactual & Actual & Simulated Mix & MP types & AP types & Confused types \\
\cline { 2 - 6 } Polynomial & -0.048 & -0.098 & -0.024 & -0.009 & -0.641 \\
& $(0.010)$ & $(0.032)$ & $(0.032)$ & $(0.029)$ & $(0.104)$ \\
2007 & -0.041 & -0.078 & -0.044 & -0.007 & -0.549 \\
\multirow{2}{*}{ 2007 Scaled } & $(0.012)$ & $(0.020)$ & $(0.016)$ & $(0.017)$ & $(0.053)$ \\
& -0.045 & -0.083 & -0.039 & -0.003 & -0.544 \\
& $(0.017)$ & $(0.021)$ & $(0.018)$ & $(0.018)$ & $(0.054)$ \\
\hline
\end{tabular}

Note: Bootstrapped standard errors shown in parentheses.

Table A5-: IV Estimates using Simulated Distributions

\begin{tabular}{|c|c|c|c|c|c|c|}
\hline \multirow[b]{2}{*}{$\Delta \ln M P$} & \multicolumn{3}{|c|}{ Actual } & \multicolumn{3}{|c|}{ Simulated Mix } \\
\hline & $\begin{array}{l}-0.136 \\
(0.007)\end{array}$ & • & $\begin{array}{l}-0.141 \\
(0.010)\end{array}$ & $\begin{array}{l}-0.133 \\
(0.008)\end{array}$ & $\cdot$ & $\begin{array}{l}-0.137 \\
(0.011)\end{array}$ \\
\hline \multirow[t]{2}{*}{$\Delta \ln A P$} & $\cdot$ & $\begin{array}{l}-0.133 \\
(0.009)\end{array}$ & $\begin{array}{c}0.010 \\
(0.013)\end{array}$ & . & $\begin{array}{l}-0.130 \\
(0.010)\end{array}$ & $\begin{array}{c}0.006 \\
(0.014)\end{array}$ \\
\hline & \multicolumn{3}{|c|}{ MP types } & \multicolumn{3}{|c|}{ AP types } \\
\hline$\Delta \ln M P$ & $\begin{array}{l}-0.131 \\
(0.002)\end{array}$ & • & $\begin{array}{l}-0.125 \\
(0.004)\end{array}$ & $\begin{array}{l}-0.080 \\
(0.003)\end{array}$ & $\cdot$ & $\begin{array}{c}0.008 \\
(0.003)\end{array}$ \\
\hline$\Delta \ln A P$ & $\cdot$ & $\begin{array}{c}-0.133 \\
(0.003)\end{array}$ & $\begin{array}{c}0.009 \\
(0.004)\end{array}$ & $\cdot$ & $\begin{array}{l}-0.135 \\
(0.003)\end{array}$ & $\begin{array}{l}-0.142 \\
(0.004)\end{array}$ \\
\hline & \multicolumn{3}{|c|}{ Confused types } & & & \\
\hline$\Delta \ln M P$ & $\begin{array}{l}-0.151 \\
(0.006)\end{array}$ & $\cdot$ & $\begin{array}{l}-0.313 \\
(0.018)\end{array}$ & & & \\
\hline$\Delta \ln A P$ & $\cdot$ & $\begin{array}{l}-0.067 \\
(0.005)\end{array}$ & $\begin{array}{c}0.240 \\
(0.020)\end{array}$ & & & \\
\hline
\end{tabular}

Note: Standard errors (clustered at premise level) shown in parentheses. 


\section{Difference in mean consumption (BCH minus NW)}
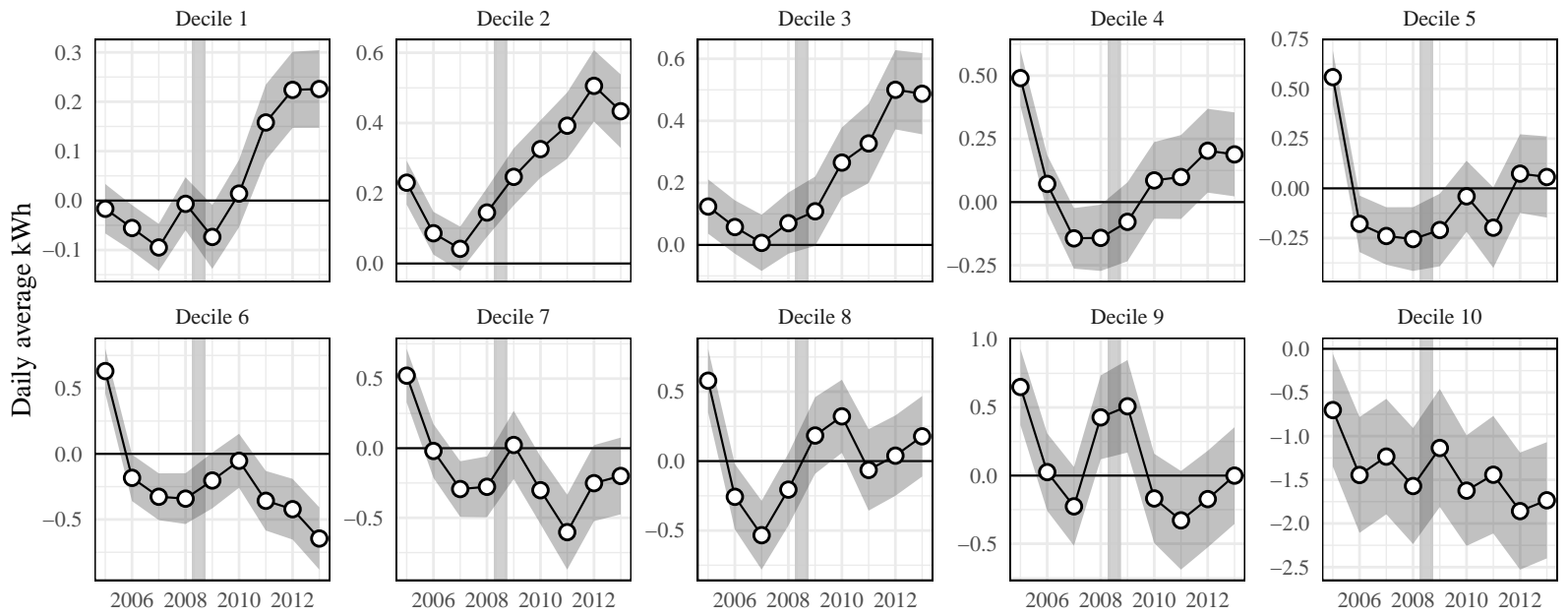

Figure A1. : Difference in MeAn COnsumpion By DECILE

Note: This plot shows the difference in mean daily consumption (in kilowatt-hours) for $\mathrm{BCH}$ minus New Westminster. Each subplot represents a different decile of the consumption distribution. Deciles are creating using all households regardless of region, thus deciles have different ratios of $\mathrm{BCH}$ vs NW. An increase in the mean consumption in $\mathrm{BCH}$ can clearly by seen in the lower deciles, whereas a fall is observed after 2008 in Decile 6. Decile 10 has consistently lower consumption in BCH vs New Westminster. This is due to a smaller number of NW households in the top decile with above decile-average consumption. 

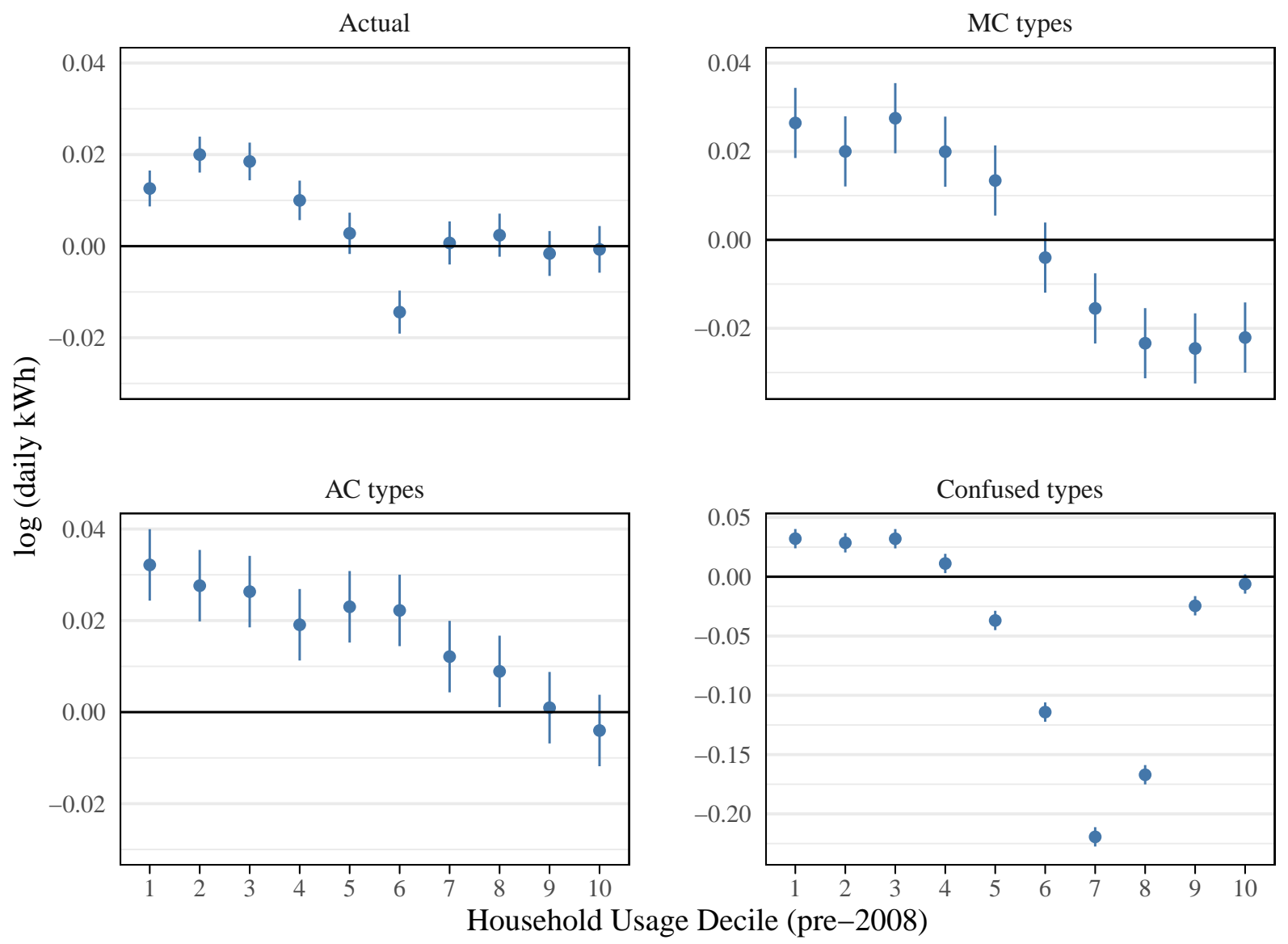

Figure A2. : Simulated Difference-IN-DIFfEREnCE COEFficients 


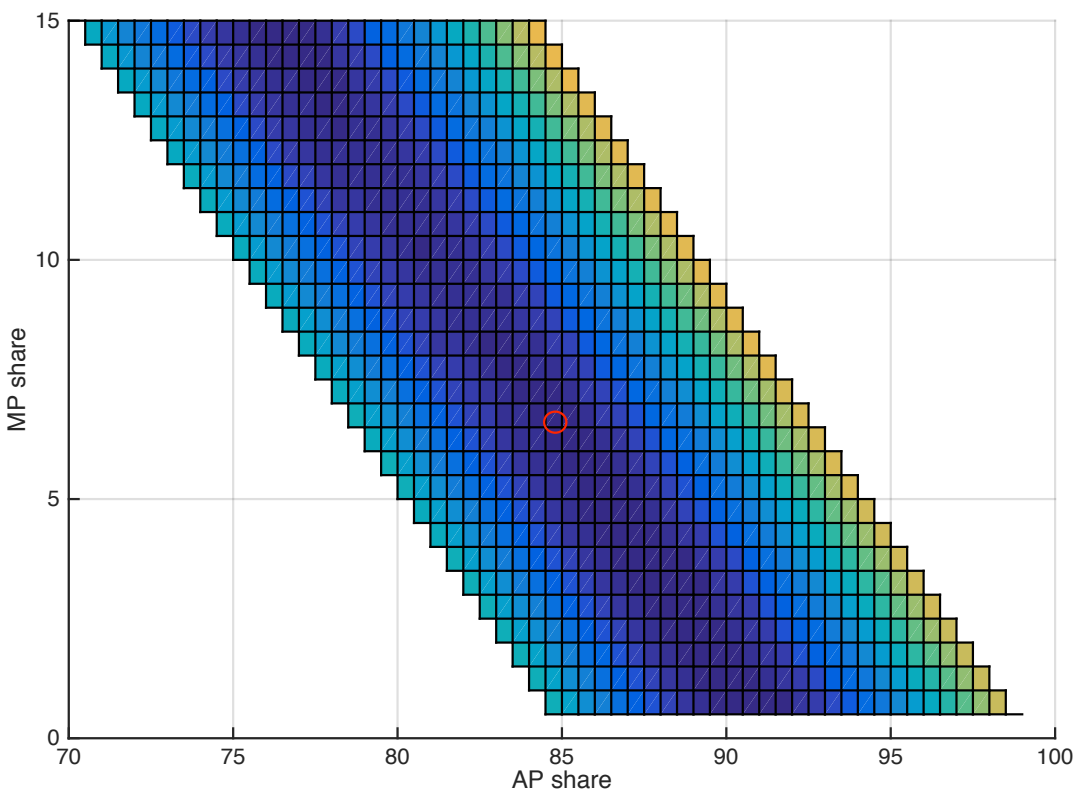

Figure A3. : PRECISION OF INDIRECT INFERENCE RESUlts

Notes: This contour plot presents the criterion function value for each iteration of repeated indirect inference for various shares of MP and AP types. The dark blue region represents the lowest values of the criterion function. The lowest point ( $85 \% \mathrm{AP}, 7 \% \mathrm{MP})$ is shown in the middle of the figure, while a "valley of minima" can be observed along the diagonal such that confused types are roughly $8 \%$. 